ring for $1000 \mathrm{~s}$ (orders of magnitude longer than typical microfluidic times). Also, the system could be operated as a filter or separator by slowly reducing the contact angle from $\theta>90^{\circ}$ to $\theta<90^{\circ}$. The capillary length scale ( $4 \mathrm{~mm}$ in the case described) was determined by the ratio of surface tension to gravitational weight. This microfluidic channel width of $4 \mathrm{~mm}$ sets the approximate upper size limit; however, as microfluidic sizes shrink, the effect becomes more efficient, and similar behavior was observed over all widths down to $100 \mu \mathrm{m}$.

SHILPA SANKHE

\section{Discretely Sized Si Nanoparticles Fluoresce in RGB Colors}

Researchers at the University of Illinois at Urbana-Champaign have demonstrated that their electrochemically etched hydrogen-capped silicon $\left(\mathrm{Si}_{n} \mathrm{H}_{x}\right)$ nanoparticles $(n>20)$ come in particular sizes (including diameters of $1 \mathrm{~nm}, 1.67 \mathrm{~nm}$, $2.15 \mathrm{~nm}$, and $2.9 \mathrm{~nm}$ ) and fluoresce in blue, green, yellow, and red, respectively, with band peaks at $\sim 410 \mathrm{~nm}, 540 \mathrm{~nm}, 570 \mathrm{~nm}$, and $600 \mathrm{~nm}$. To convert bulk silicon into nanoparticles, physics professor Munir Nayfeh and his colleagues used an electrochemical treatment that involved gradually immersing a silicon wafer into an etchant bath of hydrofluoric acid and hydrogen peroxide while applying an electrical current. The process eroded the surface layer of the material, leaving behind a network of weakly interconnected nanostructures. The wafer was then removed from the etchant and immersed briefly in an ultrasound bath.

Under the ultrasound treatment, the nanostructure network crumbled into individual particles, which could be easily separated into the different size groups. According to the authors, quantum Monte Carlo simulations indicate that the key to forming these stable configurations is the use of hydrogen peroxide in the etching solution. Although the authors do not have a full understanding yet, it appears that the hydrogen interacts with the Si and etching solution in such a way that formation of certain configurations are energetically favored. As reported in the February 4 issue of Applied Physics Letters, the silicon particles fluoresced under ultraviolet light.
They also could fluoresce when struck with two photons of infrared light, a technique that could noninvasively penetrate human tissue.

Current medical and biological fluorescent imaging is limited by the use of dye markers, which are not photostable, Nayfeh said. The dyes can break down under photoexcitation, room light, or higher temperatures. The Si particles are photostable and bright.

"By placing particles of different colors in strategic locations, you could study such phenomena as growth factors in cancer cells or how proteins fold," said Nayfeh, who also is a researcher at the UI's Beckman Institute for Advanced Science and Technology.

\section{DNA Strands Control and Fuel Robust DNA Rotary Mechanical Device}

A team of researchers at New York University has built a device from synthetic DNA molecules that improves upon previously developed nanoscale DNA devices because it allows for bettercontrolled movement within larger DNA

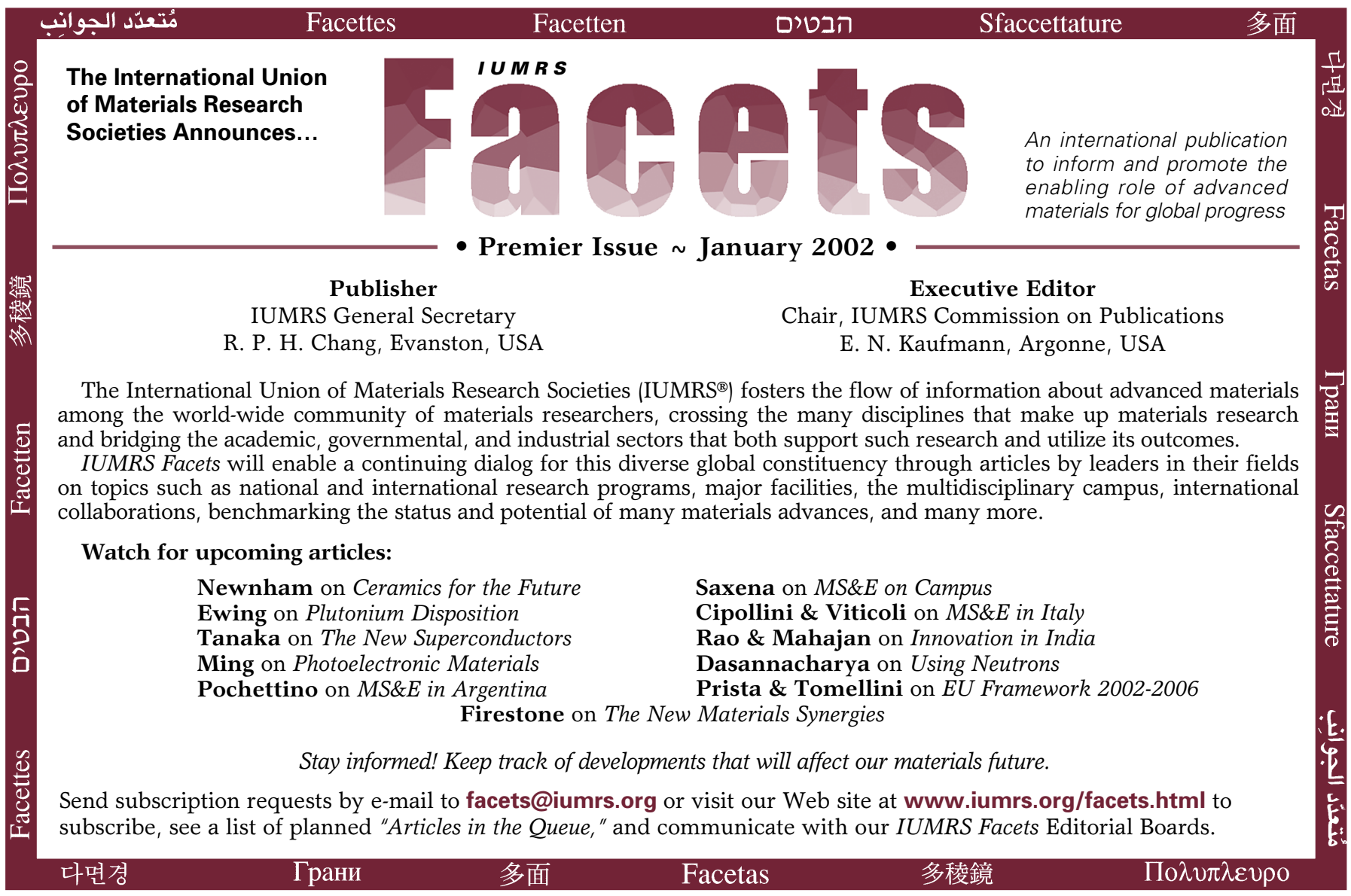


constructs. The purpose of these devices is to achieve a variety of structural configurations on the nanometer scale, thus paving the way for nanorobotics. By inserting DNA "set" strands that select the state of the device and "fuel" strands that remove the set strands to return the device to an unspecified state, into individual molecule pairs, the scientists fabricated a sequence-dependent rotary DNA device that operates in a four-step cycle.

In the January 3 issue of Nature, the scientists reported that they used paranemic crossover (PX) DNA, a four-strand molecule in which two double helices are joined by the crossing over of strands everywhere that the strands come together. The scientists produced a half-turn rotation by converting them into $\mathrm{JX}_{2}$ molecule pairs that lack two of the crossovers present in the PX structure. "Set" strands refer to the strands that position the state of the device in the PX conformation. The "fuel" strand refers to the strand that is complementary to the entire length of the set strand and will pair with it.

The scientists alternated additions and removals of fuel and set strands, holding the solution at $20^{\circ} \mathrm{C}$ for $60 \mathrm{~min}$ through each stage of the four-step cycle. Nondenaturing gel electrophoresis and atomic force microscopy verified the formation and interconversion of the PX DNA and its topoisomer $\mathrm{JX}_{2}$ DNA. The researchers report motions up to $35 \mathrm{~nm}$.

\section{Sacrificial Bonds in Collagen Contribute to Bone Strength}

With the use of atomic force microscopy (AFM), scientists at the University of California-Santa Barbara have revealed that sacrificial bonds in collagen may be partially responsible for the toughness of bone. Collagen is a protein that serves as a structural component of a variety of tissues including bone, tendon, and skin.

Using a purified cow tendon as a sample, the scientists stressed the collagen, discovering that the protein contains sacrificial bonds that rupture when stretched, then reform.

Graduate student James B. Thompson said, "These sacrificial bonds provide a mechanism for dissipating mechanical energy in collagen molecules. The time scale required for sacrificial bonds to reform in collagen correlates to the time needed for bone to recover from microscopic indentations."

Besides stretching the collagen from bones (AFM pulling using force probes with spring constants of $50 \mathrm{pN} \mathrm{nm}^{-1}$ ), the scientists made small indentations in the femur of a rat (AFM indentation with spring constants of $\sim 50 \mathrm{~N} \mathrm{~m}^{-1}$ ), discovering that the bone returns to its original shape in $\sim 30 \mathrm{~s}$. They needed a stiffer force probe to indent a bone than to stretch a collagen molecule.

As reported in the December 13 issue of Nature, the researchers noted that the sacrificial bonds found within or between collagen molecules in their samples were similar to those found in abalone shell. Thompson said it is too early to tell what impact this study will have on human health and how the study might affect technology or medicine.

\section{Carbon Nanotube Electrodes May Increase Storage of Li-Ion Batteries}

Researchers at the University of North Carolina-Chapel Hill have shown that carbon nanotubes can contain roughly twice the energy density of graphite. They suggest the possibility that more energy could be stored in batteries using carbon nanotubes than with conventional graphite electrodes.

Currently, graphite and carbonaceous materials are used as one of the two working electrodes in rechargeable Li-ion batteries. The energy capacity of the battery is partly limited by the amount of $\mathrm{Li}$ that can be stored per unit weight of carbon by the intercalation reaction. For graphite, the limit is $\mathrm{LiC}_{6}$.

Associate professor Otto Z. Zhou said, "In our experiments, we used both electrochemistry and solid-state nuclear magnetic resonance (NMR) measurements, which show similar results. With graphite, we can store, reversibly, one charged lithium ion for every six carbon atoms in graphite, but we found that with nanotubes, we can store one charged lithium ion for every three carbons, also reversibly."

As reported in the January 7 issue of Physical Review Letters, the researchers chemically etched the single-walled nanotubes (SWNTs) into much shorter cylinders. In this process, the ends of the nanotubes were opened, and the inner core spaces were exposed. The research team performed electrochemical and solid-state ${ }^{13} \mathrm{C}$ NMR experiments on both the control (purified SWNTs with closed ends) and etched SWNTs with open ends. The results showed that the reversible Li storage capacity increased from $\mathrm{LiC}_{6}$ in the closed-end SWNTs to $\mathrm{LiC}_{3}$ in the openend SWNTs.

Zhou said, "We believe this is due to $\mathrm{Li}$ diffusion into the inner core spaces of the nanotubes."

Zhou said that many technical issues must still be resolved, such as large irre- versible capacity, high intercalation voltage, and life time.

\section{Nanotube "Peapods" Exhibit Tunable Electronic Properties}

A team of researchers from the University of Illinois at Urbana-Champaign (UI) and the University of Pennsylvania have demonstrated that the encapsulation of $\mathrm{C}_{60}$ molecules in a single-walled carbon nanotube is a viable route to controlling the motion of electrons in carbon nanotubes. The samples were produced using molecular self-assembly techniques by materials science professor David Luzzi and his research group.

To explore the properties of these nanostructures-dubbed "nanoscopic peapods"-Ali Yazdani, a professor of physics at UI, and Daniel Hornbaker, a UI graduate student, used a low-temperature scanning tunneling microscope (STM), obtaining high-resolution images. As reported on the Science Express Web site on January 3 and the February 1 issue of Science, the researchers were able to image the physical structure of individual "peapods" and map the motion of electrons inside them. The researchers found that the encapsulated fullerenes modify the electronic properties of the nanotube without affecting its atomic structure.

The researchers said that the encapsulation process can in principal deform the nanotubes' structure, which is very unfavorable. Therefore, the majority of the peapods were made of nanotubes in which there was enough room to fit the $\mathrm{C}_{60}$ molecules inside. The strong electronic interaction between the $\mathrm{C}_{60}$ molecules inside and the nanotubes' electronic states was unexpected, they said.

"In contrast to unfilled nanotubes, peapods exhibit additional electronic features that are strongly dependent on the location along the tube," Yazdani said. "By mapping electron waves of different energies inside these nanoscale structures, we can begin to unravel the complex interaction in these systems and better understand their electronic properties."

Using the STM to manipulate the encapsulated molecules, the researchers compared the measurements performed on the same section of nanotube with and without the encapsulated molecules. Using tunneling spectroscopy, the researchers mapped how electronic states were distributed in energy (density of electronic states). They performed these measurements on the filled and unfilled section of a nanotube in order to compare how the electronic states of a nanotube were modified by the presence of the molecules inside. The measurements directly 